\title{
GUÍA METODOLÓGICA DE EMPODERAMIENTO ESTUDIANTIL DESDE LA GESTIÓN FORMATIVA SOCIO- CULTURAL Y PROFESIONAL EN LA UNIVERSIDAD DE TAHOUA, NÍGER
}

\section{METHODOLOGICAL GUIDE FOR EMPOWERING STUDENTS FROM THE SOCIO-CULTURAL AND PROFESSIONAL TRAINING MANAGEMENT IN TAHOUA UNIVERSITY, NIGER}

\author{
Moussa Moustapha ${ }^{1}$ \\ Marcelo Remigio Castillo Bustos ${ }^{2}$
}

Recibido: 2019-01-10 / Revisado: 2019-03-22 / Aceptado: 2019-04-01 / Publicado: 2019-07-01

Forma sugerida de citar: Moustapha, M. y Castillo-Bustos, M. R. (2019). Guía metodológica de empoderamiento estudiantil desde la gestión formativa socio-cultural y profesional en la Universidad de Tahoua, Níger. Retos de la Ciencia, 3(7), pp. 26-36. https://doi.org/10.53877/rc.3.7.20190701.03

\section{RESUMEN}

Los debates sobre la formación de profesionales en África, de manera general, y en particular en Níger, interrogan constantemente el cómo formar profesionales auténticos, competentes y comprometidos con el contexto al que pertenecen, de tal manera que sean capaces de concretar transformaciones socio-culturales y económicas efectivas. En la Universidad de Tahoua, luego de examinar los diferentes programas formativos de las carreras de ciencias de la educación, derecho y economía a través de las técnicas de observación participante, encuestas y grupos de discusión; se revelan insuficiencias relacionadas con la capacidad de los estudiantes para llevar a cabo trabajos investigativos que favorezcan las transformaciones socioculturales, en función de las necesidades contextuales. De ahí que, en este trabajo se expone una guía metodológica para potenciar las capacidades investigativas del estudiantado universitario, la aplicación de la guía permitió a los investigados enfrentar los problemas antes referidos en su entorno y proponer soluciones viables y efectivas. Los resultados demuestran avances significativos en el quehacer profesional y en su relación arraigada a su contexto de pertenencia.

Palabras clave: empoderamiento profesional, identidad, guía metodológica, desarrollo y transformación contextual.

\footnotetext{
${ }^{1}$ Doctor en Ciencias Pedagógicas (Ph.D). Profesor investigador en la Universidad de Tahoua. Niger, E-mail: mmoustapha2002@yahoo.fr

${ }^{2}$ Doctor en Ciencias Pedagógicas (Ph.D). Profesor investigador en la Universidad Central del Ecuador, E-mail: m.c.investigacion@outlook.com
} 


\section{ABSTRACT}

The debates about the formation of professionals in Africa in general but particularly in Niger are always asking about how to form authentic professionals, competent and engaged with the context that they belong to, in a way that they could be capable to concrete socio cultural and economic affective transformations. At the University of Tahoua after the review of all the different formative programs of education, legal, and economic majors through the observation techniques, surveys and focus groups, are revealed shortcomings related with the student's capacity to ideal with the researches that allow the cultural transformations, in function of the contextual necessities. Since there this project exposes the methodological guide to boost the search capacities of all the students from the university, the guide of the application allowed to the investigated people to confront the problems previously mentioned in order to propose viable and affective solutions. The results show significant advances in the professional job and in their rooted relationship to the membership context.

Keywords : Professional empowerment, identity, sociocultural, methodological guide, development and contextual transformation.

\section{INTRODUCCIÓN}

En un mundo en constante transformación, asisten múltiples exigencias que los profesionales de las distintas áreas del conocimiento tienen que enfrentar. Se requiere entonces de los profesionales una formación holística, como condición garante para su inserción en el campo laboral. En tal sentido, los programas y los procesos formativos universitarios tienen que lograr una formación profesional que desarrolle capacidades, habilidades y destrezas suficientes para actuar con pertinencia en situaciones complejas y de altas demandas.

Lo antes señalado, constituye reto de alta responsabilidad para las universidades en el contexto africano, tomando en cuenta que estas, que tienen raíces europeas y requieren indiscutiblemente contextualizarse para dar respuestas sólidas a las nuevas demandadas sociales, ya que, en la actualidad es necesario contar con profesionales que dominen los conocimientos científicos y técnicos de la disciplina de su especialización y que estén dotados de una gran capacidad metodológicas y sociales para participar con pertinencia en las transformaciones socio-culturales, económicas, políticas e ideológicas de su entorno.

\section{DESARROLLO}

El entorno de la universidad de Tahoua requiere de profesionales preparados para actuar en situaciones complejas y de alta exigencia, lo que, constituye un reto para las universidades de la Unión Económica Monetaria Oeste Africana, ya que deben dar respuesta a las exigencias sociales a través del desarrollo de nuevas capacidades en los profesionales que están formando. Cabe precisar que, en la actualidad se requieren profesionales con sólidos conocimientos científicos y técnicos, pero que además sean poseedores de capacidad metodológica, social y participativa, de tal suerte que interactúen con pertinencia en la realidad, a la vez, que resuelven los problemas de su quehacer cotidiano.

Para desarrollar las referidas capacidades, es vital articular metodologías formativas en las que los estudiantes se constituyan en actores principales, 
enfrentado situaciones reales o simuladas vinculadas con su futuro quehacer profesional. De ahí que, la práctica formativa profesional constituye un espacio curricular y extracurricular que agrega valor a la formación de los estudiantes y que facilita el proceso de orientación e inserción en el campo laboral, ya que permite aplicar en contextos laborales reales los conocimientos científicos y técnicos, constituyéndose así, en un puente de suma importancia entre el campo laboral y el académico.

En tal sentido, si la misión de la Universidad de Tahoua, es la formación de un profesional con capacidad de transformar su contexto socio-histórico-cultural de pertenencia, surgen entonces las siguientes interrogantes: ¿qué fundamentos epistemológicos?, ¿qué metodología? y ¿qué métodos y procedimientos?, deben ser empleados para alcanzar un verdadero desarrollo sociocultural, en concordancia con los planteamientos anteriores.

Téngase en cuenta que, ser profesional de y para un contexto como el de la Universidad de Tahoua, significa conocer y apropiarse de sus tradiciones, costumbres, cosmovisión e imaginario socio-cultural, que al decir del filósofo escocés Hume (1739), esos aspectos constituyen la guía fundamental de la vida humana, destacando que son efectivamente conocimientos que suponen una guía importante para comprender el alma de un país y de su gente.

En tal circunstancia, una formación profesional que toma las realidades socioculturales del contexto de la Universidad de Tahoua constituye no solo la vía expedita para alcanzar las aspiraciones y anhelos de los estudiantes y de sus pueblos, sino una necesidad y una alternativa para formar a profesionales comprometidos con el bienestar de la sociedad.

Lo anterior, ha de obligar a elaborar una metodología que ha de permitir llevar a cabo tal proceso formativo profesional en el espacio de la Universidad de Tahoua, lo que constituye una herramienta de empoderamiento formativo profesional en ese contexto universitario, no solo por la integración holística y dialéctica de la cultura científica, los saberes tradicionales locales, las costumbres, los valores socioculturales contextuales, profesionales que los actores involucrados han de comprender, apropiarse, sistematizar y generalizar mediante la participación e interacción profesional, en pos de construir nuevos conocimientos científicos que permitan la continuidad y la consecutividad del proceso otorgándoles la oportunidad de tomar decisiones y de orientar hacia nuevos y continuos procedimientos de desarrollo, transformación y de innovación científica.

La metodología propuesta, inquiere una práctica formativa contextualizada, que ha de sustentarse en el modelo de gestión formativo socio-cultural profesional en las universidades de la Universidad de Tahoua, cuyo supuesto reivindica por una parte, una formación profesional que toma en cuenta las verdaderas problemáticas del contexto, en relación dialéctica con la apropiación socio-cultural de la profesión como medio de adquisición de conocimientos científicos que permiten a los estudiantes rescatar, crear y transformar los problemas contextuales y profesionales.

Ello, es posible llevando el proceso a un nivel superior de desarrollo y transformación, mediante la autenticidad formativa profesional, como proceso de auto-transformación, auto-reafirmación y de solución a las necesidades y problemas reales del contexto donde el empoderamiento formativo profesional se erige como factor de creación, continuidad y consecutividad, desde una intencionalidad formativa basada en la participación y la toma de decisiones. 
En la literatura especializada, abundan acepciones del concepto metodología. Hay autores como Coelho (2019), quien la considera como una serie de métodos y técnicas de rigor científico que se aplican sistemáticamente durante un proceso de investigación para alcanzar un resultado teóricamente válido. En este sentido, la metodología funciona como el soporte conceptual que rige la manera en que se aplican los procedimientos en una investigación.

La enciclopedia colaborativa en la red cubana EcuRed (en línea), define a la metodología como la parte de la lógica, cuya finalidad es señalar el procedimiento para alcanzar el saber de un orden determinado. La metodología es entonces parte del proceso de investigación, que sigue a la propedéutica y permite sistematizar los métodos y las técnicas necesarias a emplear en tal proceso. Los métodos elegidos por el investigador facilitan el descubrimiento de conocimientos válidos y confiables, potencialmente utilizables en la solución de los problemas.

Matos y Cruz (2011), consideran que la metodología es "un conjunto de métodos, procedimientos y técnicas que responden a cada objeto investigado en relación con sus características y fundamentos epistemológicos. De ahí que, expresan la lógica de actuación didáctico-metodológica donde se imbrican objetivo, contenido, método, evaluación, etc., demostrando su interrelación y sistematización en el proceso construido", concepto que se asume en este trabajo.

Así, la guía metodológica de empoderamiento estudiantil desde la gestión formativa sociocultural y profesional en la Universidad de Tahoua se caracteriza por ser flexible, ya que está abierta a las experiencias de otros procesos similares que pueden contribuir a su perfeccionamiento, valorando siempre las realidades socioculturales del contexto africano, que permiten satisfacer las necesidades profesionales y contextuales. Asimismo, tiene un carácter participativo, ya que propicia su enriquecimiento mediante la reflexión y el debate colectivo en un clima de comunicación abierta.

La metodología es sistémica, por tener relaciones que interactúan unas con otras. Es integradora, ya que desde el punto de vista teórico-metodológico, toma en cuenta los referentes que influyen en la solución de los problemas científicos, y las categorías del proceso en su relación con los intereses culturales, profesionales y sociales. Es también, desarrolladora porque ha de permitir la socialización de los resultados alcanzados, comparando la solución dada a los problemas profesionales contextuales por los estudiantes, de manera que se produzcan cambios cualitativos en los mismos.

Otro aspecto que caracteriza a la metodología para la gestión formativa sociocultural profesional en las universidades de la Unión Económica Monetaria Oeste Africana, es la manera asequible para lograr su aplicación, concebida como herramienta pedagógica para facilitar la práctica formativa profesional en el contexto de la Universidad de Tahoua. Los métodos y procedimientos son prácticos y flexibles de modo general, de ahí, su asequibilidad.

\section{Objetivo de la metodología}

La metodología tiene como objetivo el logro de un empoderamiento formativo profesional, a través de la práctica formativa sociocultural profesional contextualizada, lo que otorga al proceso, una autenticidad formativa profesional en la Universidad de Tahoua. Ello permitirá a los estudiantes una interacción profesional con las realidades socioculturales de su contexto, promoviendo el desarrollo de capacidades y habilidades profesionales para interpretar los fenómenos, hechos y sucesos del contexto, a la vez que, construyen sentidos y 
significados que les comprometen en la solución de los problemas profesionales y contextuales.

Así, para la concreción práctica de la metodología para la gestión formativa sociocultural profesional en la Universidad de Tahoua, se elabora métodos para profesores, estudiantes y directivos, los mismos guardan una relación dialéctica con el modelo del profesional competente. Los métodos elaborados se estructuran en procedimientos que fomentan una interacción entre estos actores involucrados, permitiéndolos a cada uno, desempeñar el rol que le corresponde para la ejecución de la práctica formativa de empoderamiento.

Los métodos elaborados, ponen énfasis en las diferentes actividades que propician el desarrollo de las capacidades profesionales de los actores involucrados, particularmente los estudiantes, a quienes están dirigidos, estimulando su participación en proyectos de investigación sobre las problemáticas reales del contexto.

\section{Métodos para los profesores}

En la formación de los estudiantes, el profesor es fundamentalmente mediador del aprendizaje entre los contenidos y la actividad constructivista del estudiante. Toda la intervención docente se orienta a conseguir que los estudiantes se conviertan en personas autónomas, competentes para responder de manera eficaz y diligente a los cambios y versiones que le ofrezcan los contextos en los que interactúan.

El profesor en el proceso de empoderamiento formativo desde su especialidad, ha de contribuir en la formación de seres humanos estratégicos, lo cual, permite potenciar la actuación profesional de los estudiantes de manera que cada uno, pueda elaborar un modelo personal de sus acciones. De ahí que, para garantizar su alcance, se precisa necesario que los profesores conozcan:

- Los conceptos teóricos del conocimiento específico;

- Los procedimientos para identificar, problematizar y resolver los problemas teóricos y metodológicos;

- Saber estimular a los estudiantes en la búsqueda de información especializada en revistas, folletos, tesis, internet, libros y otros medios;

- Saber motivar a los estudiantes a aclarar las dudas durante y después de cada sesión de trabajo;

- Saber promover la creación y el pensamiento divergente;

- Saber promover la utilización de las tecnologías de la información y la comunicación (TIC) en los procesamientos de la información y la elaboración de los informes investigativos;

- Saber mantener un control sistemático durante y después de cada actividad.

Los profesores como guías de la implementación metodológica para la gestión formativa sociocultural profesional y de empeoramiento estudiantil, deben atender también los siguientes elementos metodológicos:

- La atención sistemática de los estudiantes, individualmente y colectivamente como manera de corregir sus insuficiencias para el desarrollo de sus capacidades profesionales y transformadoras;

- Los problemas del contexto deben ser discutidos profundamente de modo que sean los de mayor nivel de generalización y sistematización;

- Orientar algunos procedimientos y métodos necesarios para reconocer, plantear y resolver los problemas de las realidades socioculturales;

- Fomentar la auto-interrogación que habitúa a los estudiantes a convertir sus conocimientos en objeto de análisis y a cuestionar su propio pensamiento; 
- Orientar adecuadamente para que puedan realizar la elección consciente, de los métodos y procedimientos de trabajo, las técnicas y recursos empleados, así como, la debida utilización del tiempo;

- Favorecer el desarrollo de la argumentación en foros de exposición y debate con otros o consigo mismo.

\section{Procedimientos de los Métodos para los profesores, para ser aplicados en la metodología}

- Caracterizar a partir de diagnóstico las particularidades socioculturales de los estudiantes;

- Diagnosticar mediante talleres de intercambios con los estudiantes, el nivel de conocimientos que tienen los mismos sobre los diferentes aspectos teóricometodológicos de su carrera y las realidades desde varias aristas del contexto;

- Profundizar en los aspectos socio-culturales importantes del contexto que contribuyen al desarrollo de identidad del profesional;

- Rememorar los diferentes aspectos específicos de la carrera que permiten comprender la realidad social y cultural del contexto;

- Abordar algunas teorías y metodologías de investigación científica socioeducativa para recapacitar a los estudiantes;

- Seleccionar los aspectos teórico-metodológicos que han de ser apropiados de manera intencional por los estudiantes, los cuales deben de constituirse en los objetivos y los proyectos formativos profesionales de la universidad;

- Valorar los diferentes fenómenos, hechos y sucesos que han influido en el contexto socio-histórico-cultural;

- Desarrollar en los estudiantes, capacidades y habilidades de trabajo colectivo mediante prácticas investigativas socio-culturales en instituciones o centros socio-culturales del contexto y de la región;

- Orientar y guiar los estudiantes de manera individual, en las búsquedas de los conocimientos socio-culturales que propicien su desarrollo de identidad y profesional al contexto de pertenencia;

- Promover, estimular debates y críticas científicas sobre las realidades socioculturales del contexto universitario en los estudiantes;

- Dictaminar los principales logros e insuficiencias en el proceso.

\section{Métodos para los estudiantes.}

El rol de los estudiantes en el proceso formativo de empoderamiento es fundamentalmente de responsabilidad y de autorregulación de su proceso de aprendizaje. La autorregulación es la capacidad auto-reflexiva que consiste en la inclinación de los estudiantes a pensar o reflexionar sobre ellos mismos, y sobre distintas alternativas, de tal manera, que configuran su acción en función de satisfacer sus necesidades, a pesar de las condicionantes de su entorno.

Los estudiantes deben abordar de modo autónomo y estratégico el aprendizaje que consideran significativo para la creación de nuevos conocimientos, como proceso y resultado de su desarrollo continuo.

En este proceso formativo sociocultural profesional de empoderamiento de los estudiantes universitarios, los estudiantes, constituyen el eje de desarrollo de dicho proceso, ya que todo se centra en la búsqueda del desarrollo de sus capacidades profesionales y de identidad con el contexto. Para ello, deben de tener conocimientos teórico-metodológicos básicos y consolidados de su profesión, de tal manera que les permitan un acercamiento a los fenómenos, hechos y sucesos del contexto. 
Así con una actitud básica de responsabilidad y una capacidad de iniciativa, los estudiantes deben:

- Saber identificar sus necesidades de formación en relación con el contexto socio-histórico-cultural que requieren una permanente actualización y evaluación sustentada en la elaboración de proyectos personales y profesionales, de sí mismos como referente de sus itinerarios formativos profesionales;

- Ser conscientes del perfil de competencia concreta que quieren y aspiran alcanzar, realizando un análisis de las actitudes y valores, habilidades y destrezas que van desarrollando, y a partir de ahí, exponer las carencias y necesidades formativas detectadas en su proceso reflexivo;

- Saber establecer sus objetivos de aprendizaje. De este modo la claridad de los objetivos visualizados que son de competencias profesionales que guarden relación con el contexto socio-histórico-cultural, les ayudará a definir el tipo de aprendizaje que deben llevar a cabo y los criterios de evaluación de sus logros;

- Generar confianza en sí mismos, desarrollando sentimientos de autoeficacia que permitan la formulación de expectativas de éxito;

- Saber encontrar, seleccionar, contrastar y procesar la información pertinente al objeto de estudio y a las competencias que se quieren desarrollar;

- Saber establecer con su tutor un proceso de supervisión de su propio aprendizaje, concretado en sesiones presenciales y/o en comunicaciones mediante las Tecnologías de la Información y las Comunicaciones;

- Saber gestionar los éxitos y errores, identificando las claves de unos y otros;

- Organizar portafolios que recojan de forma sistemática y ordenada las evidencias del aprendizaje autónomo realizado, acompañado de sus reflexiones sobre el itinerario seguido.

\section{Procedimientos de los métodos para los estudiantes para ser aplicados en la metodología}

- Apropiarse de los hábitos, valores y habilidades conociendo los modos de actuación de su profesión;

- Comprender, explicar e interpretar los acontecimientos, sucesos históricosocioculturales del contexto y su incidencia en la región;

- Valorar las tradiciones histórico-culturales que se fueron trasmitiendo de generación en generación y que constituyen un avatar para el proceso formativo profesional en el contexto;

- Valorar e identificar las diferentes manifestaciones de la identidad sociocultural en el contexto;

- Sistematizar los conocimientos científicos y socioculturales del contexto;

- Elaborar informes en cada etapa del objeto investigado.

\section{Métodos para los directivos}

El campo de intervención pedagógica de los directivos como conductores del proceso, lleva acciones que involucran a los diversos sujetos que interactúan en los centros educativos. Su caracterización es compleja y demanda la construcción de herramientas conceptuales que permitan comprender la realidad institucional y contextual, así como desarrollar las capacidades para operar sobre esta. La noción del rol de los directivos, se refiere a un conjunto organizado de comportamientos correspondientes a un oficio o a un puesto de trabajo determinado que abarca una serie de actuaciones y cumplimiento tareas. 
El papel de los directivos en este proceso de desarrollo y transformación, es facilitar las condiciones necesarias para la concreción de la metodología como práctica formativa sociocultural profesional en las comunidades, instituciones 0 centros socioculturales del país o de la región. En tal circunstancia, han de establecer relaciones que estructuran el proceso, los encuentros y su desarrollo, organizando así la participación de los estudiantes y profesores para el cumplimiento de los objetivos, en equipo. Además de coordinar, supervisar y evaluar las prácticas formativas en el campo y su incidencia social, los directivos deben de promover y motivar a los estudiantes hacia la participación activa.

Para un buen desempeño de su rol en este proceso, los directivos deben de:

Saber propiciar que las actividades desarrolladas por los estudiantes sean congruentes con el entorno. El éxito de todo proceso depende de la supervisión y coordinación del director;

- Saber impulsar, promover y facilitar el cambio y la innovación en los centros de formación;

- Saber crear y mantener una cultura propia que permita dar sentido a la actividad que realizan los estudiantes, ayudándoles a asumir los valores, normas y objetivos de la universidad y la implicación crítica necesaria para alcanzarlos.

\section{Procedimientos del método para los directivos para ser aplicados en la metodología}

- Saber crear y mantener una cultura propia que permita dar sentido a la actividad que realizan los estudiantes, ayudándoles a asumir los valores, normas y objetivos de la universidad, y analizar las diferentes transformaciones que se manifiestan en los estudiantes, profesores y contextos;

- Lograr una retroalimentación constante entre profesores y estudiantes donde ambos se complementen y contribuyan al crecimiento profesional y personal;

- Crear y propiciar espacios de debate y de valoración científico-socio-cultural (seminarios, mesas redondas, foros sociales, eventos científicos) para el desarrollo profesional y la sistematización de las experiencias en el proceso;

- Integrar los objetivos instructivos y educativos como formación de la identidad socio-cultural en los estudiantes universitarios;

- Determinar nuevos objetivos formativos en función del desarrollo del proceso de gestión formativa socio-cultural profesional;

- Evaluar el sentido ético-profesional en el desarrollo personal, profesional y contextual que oriente la construcción de nuevos conocimientos socioculturales profesionales;

- Promover talleres, foros científicos, concursos, mesas redondas sobre las realidades socioculturales del contexto para su formación profesional.

Evaluación y patrones para corroborar los resultados alcanzados

La evaluación, como parte integrante del proceso de formación profesional y como medio facilitador de la regulación del aprendizaje y de la construcción de conocimiento, permite la comprobación y el dominio del contenido que corresponde a los docentes, estudiantes y directivos. Lo que significa que la valoración del grado de desarrollo y satisfacción, ha de ser alcanzado y manifestado en los diversos niveles de aprendizaje y sobre todo, en el grado de dominio alcanzado.

La evaluación de las acciones desarrolladas durante la aplicación de la metodología, constituye también un medio de retroalimentación que permite la corroboración de los niveles de desarrollo alcanzados en el proceso de gestión 
formativa sociocultural profesional en la universidad de Tahoua, desde una visión integral, holística y dinámica para corregir las insuficiencias detectadas en el proceso para su fortalecimiento.

Evaluación de los profesores: Nivel de gestión metodológico para la concreción práctica formativa sociocultural profesional en contexto.

\section{Patrones de logros}

- Constatar la pertinencia de las diversas propuestas formativas de los estudiantes;

- Evidenciar la presentación de los problemas socioculturales reales en los que confluyen perspectivas disciplinares complementarias;

- Constatar la problematización y cuestionamiento de la realidad haciendo que surjan preguntas estimulantes que ayuden a los estudiantes a darse cuenta de que hay conocimientos que no tienen y que les interesaría comprender;

- Evidenciar la realización de feed-back (opiniones) a los sucesivos temas y proyectos investigativos de cada estudiante a lo largo de toda la práctica formativa;

- Constatar la correspondencia de las acciones formativas en el desarrollo de una identidad formativa en los estudiantes participantes;

- Evidenciar la pertinencia de las estrategias y procedimientos elaborados que permiten una buena orientación de los estudiantes en la práctica formativa socio-cultural profesional en el contexto.

Evaluación de los estudiantes: Nivel de desarrollo de capacidades valorativas socio-cultural profesional alcanzado.

\section{Patrones de logros:}

- Evidenciar el nivel de responsabilidad de los estudiantes;

- Comprobar la elaboración de informes de la práctica formativa y el nivel de profundad en la interpretación del objeto investigado;

- Comprobar la capacidad del estudiante de trabajar en grupo;

- Comprobar el nivel en el desarrollo de habilidades y éticas profesionales en los estudiantes;

- Evidenciar el nivel de la capacidad valorativa científica sobre los diferentes aspectos socio-culturales del contexto africano;

- Verificar el nivel de creatividad en la práctica formativa de los aspectos socioculturales investigados;

- Constatar la capacidad de expresión oral de los estudiantes durante la defensa de sus informes investigativos individuales y grupales;

- Examinar la profundad de la propuesta (estrategias, proyectos) elaborada por los estudiantes para la solución del objeto investigado;

- Apreciar el nivel de generalización y sistematización de los conocimientos científicos y socio-culturales contextuales adquiridos.

Evaluación de los directivos: Nivel de responsabilidad directiva, flexibilidad, compromiso de transformación y desarrollo contextual.

Patrones de logros

- Comprobar el nivel de efectividad en la planificación, organización, ejecución y control de la práctica formativa profesional;

- Evidenciar el cumplimiento de los objetivos pedagógicos y didácticos de la práctica formativa profesional;

- Valorar el nivel de satisfacción de los actores involucrados, respecto a la coordinación del proceso; 
- Apreciar el nivel de satisfacción de los estudiantes respecto a su preparación profesional, en la práctica formativa profesional;

- Constatar el establecimiento de relaciones directas y fructíferas de los directivos con los profesores y estudiantes;

- Comprobar la fluidez de la comunicación de los directivos con los actores involucrados;

- Evidenciar la flexibilidad en la dirección de la práctica formativa profesional en contexto;

- Comprobar nivel de efectividad en la planificación, organización, ejecución y control de la práctica formativa profesional.

\section{CONCLUSIÓN}

Teniendo en cuenta que la Universidad de Tahoua, es una institución universitaria joven creada en el 2010 y con amplias expectativas en el orden académico y científico, la guía metodológica de empoderamiento de los estudiantes constituye una vía que pone a los estudiantes en contacto con el contexto universitario en función de promover cambios y transformaciones significativas; a la vez, permite desde la teoría y la práctica, desarrollar capacidades, valores, habilidades, hábitos, destrezas y éticas profesionales en los estudiantes escogidos.

Para la concreción de la guía, se elaboró un conjunto de métodos que involucran a todos los actores institucionales (estudiantes, profesores y directivos), por el rol diferenciado que desempeñan en el proceso de empoderamiento de los estudiantes. Los métodos usados se estructuran en procedimientos que sustentan y viabilizan desde una gestión formativa sociocultural hacia el empoderamiento de las capacidades profesionales del estudiantado. Asimismo, para el cumplimiento de los objetivos, se elaboraron un conjunto de patrones de logros que permiten la evaluación de los actores involucrados, sobre todo, los estudiantes, lo cual, permitió constatar un salto cualitativo en el desarrollo humanístico y profesional de los estudiantes.

\section{RFERENCIAS BIBLIOGRÁFICAS}

Álvarez de Zayas, Carlos (1999): "Proceso de formación de profesionales. La Escuela en la Vida". Editorial Félix Varela, La Habana, Cuba

Association des Universités Africaines (2006). Initiative régionale de mobilisation de capacité (IRMC) pour la revitalisation de l'enseignement supérieur en Afrique. Accra, Ghana. (soporte magnético)

Baczko, Bronislaw (1984). Los imaginarios sociales. Memorias y esperanzas colectivas, Edición Nueva Visión, Buenos Aires, Argentina

Banque Mondiale (1995). Maintenir la capacité d'enseigner dans des universités africaines: Problèmes et perspectives. Région-Afrique. (soporte magnético)

Benaoum Mohiédine (2011). Le système LMD: Perception des étudiants de première annéeÉvaluation de l'existant et nécessité d'un accompagnement plus approprié. Revue Synergies, Alger, Algérie n 12 del 2011

Castoriadis, Cornelius (1975). La institución imaginaria de la sociedad. RevistaTusquéts 2 Vol. Buenos Aires, Argentina

Coelho, F. (2019). "Metodología de la investigación". En: Significados.com. Disponible en: https://www.significados.com/metodologia-de-la-investigacion/ Consultado: 31 de agosto de 2019, 05:57 pm. 
Didriksson, A. La construcción de una estrategia de transformación Educativa y Universitaria. Primer Congreso. Distrito Federal de México. En http:// www.df.gob.mx/virtual/altedu/3.html. (Consultado en febrero 2011).

Estrabao, Alejandro (2002). Modelo para la gestión de los procesos de pertinencia e impacto en las facultades universitarias en la Universidad de Oriente. Tesis presentada en opción al grado científico de Doctor en Ciencias Pedagógicas. Centro de Estudios de Educación Superior "Manuel F. Gran", Universidad de Oriente, Santiago de Cuba.

Fuentes H.; Estrabao, A.; Macias T. (2003). La Universidad y su gestión: una mirada dialéctica holística. Santiago de Cuba, CeeS Manuel. F. Gran, Universidad de Oriente.

Fuentes Homero; Jorge Montoya; Liana Fuentes (2009). Los paradigmas de la ciencia y la investigación científica. CEES "Manuel F. Gran". Universidad de Oriente, Santiago de Cuba.

Fuentes, H. (2008). La formación de los profesionales en la contemporaneidad. Santiago de Cuba: CeeS Manuel. F. Gran.

Galicia Segura, Graciela (2005). La Formación de la Identidad y la Orientación Educativa en la Perspectiva Narrativa de Brune. Revista Mexicana de Orientación Educativa, No 4.

González Fernando, Rey (1989). Personalidad, su educación y desarrollo. Editorial Pueblo y Educación. Ciudad la Habana, Cuba.

Guadarrama González, P. (1990). Lo universal y lo específico en la cultura. Editorial Ciencias Sociales, La Habana, Cuba

Hart Dávalos, A.: La cultura y la formación del hombre nuevo. Revista Honda de la Sociedad Cultural José Martí. Número 8 del 2003

Horruitiner Pedro, Silva (2006). La universidad cubana: El modelo de formación. Editorial Félix Várela, Habana, Cuba

Ki-zerbo, Joseph (1990). Éduquer ou périr. Édité par I'UNICEF aux Éditions Harmattan, Paris. France

Moumouni, Abdou (1964). L'Éducation en Afrique. Edition François Maspero, Paris, France

Niang, S. (1998). Les universités africaines et la mondialisation. Dans Enseignement Supérieur en Afrique: Réalisations, défis et perspectives, Dakar. Sénégal (soporte magnético).

Sánchez Capdequí, Celso (1999). Imaginación y sociedad. Una hermenéutica creativa de la cultura. Tecnos-Universidad Pública de Navarra, Madrid. España

Tünnermann, C. (2009). La educación superior necesaria para el siglo XXI. En Revista Temas no. 57 enero-marzo.

Wandira, A. (1977). The African University in Development. Edition Ravan. Johannesburg, South Africa. 